Original research article

\title{
Correlation of adenosine deaminase operating under nitro- oxidative stress with tumor and vascularization in patients with advanced gallbladder carcinoma
}

\author{
Nabila Tounsi ${ }^{1}{ }^{*}$, Bahia Djerdjouri ${ }^{1}$, Chafik Bouzid ${ }^{2}$, Kamel Bentabak ${ }^{2}$ \\ ${ }^{1}$ University of Sciences and Technology Houari Boumediene, Faculty of Biological Sciences, Laboratory of Cellular and Molecular Biology, Algiers, Algeria \\ ${ }^{2}$ Mustapha Pacha Hospital, Public Health Center Pierre and Marie Curie, Department of Oncological Surgery A, Algiers, Algeria
}

\begin{abstract}
This study investigates serum redox status and adenosine catabolism markers in relation to tumor and angiogenesis, in patients with gallbladder carcinoma (GBC).

The level of adenosine deaminase (ADA) and xanthine oxidase $(\mathrm{XO})$ activities, nitrites $\left(\mathrm{NO}_{2}^{-}\right)$, glutathione (GSH) and malondialdehyde (MDA) were measured in sera of $40 \mathrm{GBC}$ patients and 40 healthy donors. In parallel, 15 tumors at TNM stage IV were scored for CD34 expression and microvessel density (MVD).

The results showed that XO and ADA activities, nitrites and MDA levels enhanced by $1.26(p<0.01), 2.69,2.0$, and 3.2-fold ( $p<0.001)$, respectively, while those of GSH decreased by $44.6 \%(p<0.001)$. According to receiver operating characteristic (ROC) curve, the optimal cut-off for XO, ADA, MDA, GSH and nitrites were 5.41U/1, 17.02 U/1, 3.72 $\mu \mathrm{M}, 36.91 \mu \mathrm{M}$ and $21.21 \mu \mathrm{M}$, respectively. Spearman correlation revealed that ADA activity correlated to nitrites levels $(r=0.3419, p<0.05)$ and XO activity $(r=0.5487, p<0.001)$. Multivariate binary logistic regression analysis revealed that MDA $(\mathrm{OR}=5.78, p<0.05)$, ADA $(\mathrm{OR}=1.28, p<0.001)$ and $\mathrm{XO}(\mathrm{OR}=2.81, p<0.05)$ correlated positively to GBC. CD34 was up expressed in $73.3 \%$ of tumors at intermediate to high levels. Multiple regression analysis showed that ADA affected MVD $(r=0.604, p<0.01)$.

The results suggest that high MDA/GSH ratio is a potential biomarker of GBC. In addition, the oxidative adenosine catabolism indicated that active purine salvage pathway could support tumor progression by sustaining angiogenesis.
\end{abstract}

Keywords: Adenosine deaminase; Gallbladder adenocarcinoma; Microvessel density; Nitro-oxidative stress; Xanthine oxidase

\section{Highlights:}

- Adenosine deaminase (ADA), xanthine oxidase (XO) and nitrites increased in GBC;

- ADA and XO activities correlated with GBC;

- ADA activity supported microvessel density (MVD) in advanced GBC;

- High serum MDA/GSH ratio as potential risk factor in GBC.

\begin{abstract}
Abbreviations:
ADA, adenosine deaminase; Adk, Adenocarcinoma; Ado, adenosine; AJCC, American Joint Committee on Cancer; AUC, area under the curve; CD34, cluster of differentiation 34; GBC, gallbladder carcinoma; IL, interleukin; INF $\gamma$, Interferon gamma; iNOS, inducible nitric oxide synthase; MVD, microvessel density; NO, nitric oxide; ROC, receiver operating characteristic; Th17, T helper 17; TNM, tumornode metastasis; TNF- $\alpha$, tumor necrosis factor alpha; VEGF, vascular endothelial growth factor.
\end{abstract}

\section{Introduction}

Gallbladder carcinoma (GBC) is the 20th most occurring cancer, worldwide. This lethal malignancy with a median survival expectancy of less than 1 year, ranks the 5th global incidence as a cancer of gastrointestinal tract. Its annual incidence $(2.2 / 100,000)$ and mortality $(1.7 / 100,000)$ vary with ethnicity and geographical localization (Hundal and Shaffer, 2014; Sharma et al., 2017; Shukla et al., 2018).
The poor prognosis of GBC comes from its silent progression with rapid spread and metastasis. To date, the complete resection of the gallbladder remains the only curative approach.

Chronic gallbladder inflammation-associated cancer has various etiologies, including genetic susceptibility through hyper methylation of several genes, a long time exposure to environmental pollutants, chronic infections, as well as obstruction of bile ducts by pancreatico-biliary junctions, gallstones or polyps (Ferlay et al., 2015; Tekcham and Tiwari, 2016).

\footnotetext{
* Author for correspondence: Nabila Tounsi, BP 32, El-Alia 16111, Bab-Ezzouar, Algiers, Algeria; e-mail: analib77@yahoo.fr http://doi.org/10.32725/jab.2019.016

Submitted: 2019-03-14 • Accepted: 2019-08-20 • Prepublished online: 2019-09-17

J Appl Biomed 17/3: 175-183 • EISSN 1214-0287 • ISSN 1214-021X

(c) 2019 The Authors. Published by University of South Bohemia in České Budějovice, Faculty of Health and Social Sciences.

This is an open access article under the CC BY-NC-ND license.
} 
Gallstones were diagnosed in most of GBC patients, and considered as a major risk factor (Espinoza et al., 2016; Sharma et al., 2017).

Persistent nitro-oxidative stress gives rise to gallbladder inflammation, which could shift chronic cholecystitis into gallbladder carcinoma (Cipak et al., 2017; Espinoza et al., 2016; Waris and Ahsan, 2006). Indeed, up regulation of inducible nitric oxide synthase (iNOS)-releasing nitric oxide (NO), a precursor of reactive oxygen/nitrogen species (ROS) was associated with poor prognosis for cancers of variable origins, including gallbladder (De Oliveira et al., 2017; Thompson et al., 2015). Moreover, gallbladder tumor aggressiveness was associated with chronic stromal iNOS acivity (Niu et al., 2004; Zhang et al., 2003).

The ROS-dependent oxidation of polyunsaturated fatty acids scaffolding membrane lipid bilayer, releases lipid peroxides such as 4-hydroxynonenal (HNE) and malondialdehyde (MDA). These volatile electrophiles bind to DNA and proteins generating stable toxic adducts that contribute to alteration of membrane permeability, mitochondrial dysfunction, apoptosis and cell proliferation (Cipak et al., 2017; Gasparovic et al., 2017; Zhong and Yin, 2015). Their neutralization by thiol-conjugation depletes glutathione (GSH) stores, and alters the protective antioxidant network at both cellular and systemic level (Ramsay and Dilda, 2014; Singhal et al., 2015).

Adenosine deaminase (ADA), an ubiquitous enzyme of purine salvage pathway, binds to the cell surface anchoring protein CD26, and hydrolyses circulating adenosine (Ado) to inosine and hypoxanthine. The latter is sequentially oxidized to xanthine and to uric acid by the rate limiting enzyme of purine catabolism, xanthine oxidase (XO). Importantly, $\mathrm{XO}$ is actively involved in drugs detoxification and in metabolic activation of carcinogens (Battelli et al., 2016). Ado exerts a paracrine anti-inflammatory effect on lymphoid and on myeloid cells (Kepp et al., 2017; Whiteside, 2017). Ado uptake from intra and extracellular pools provides ATP and nucleotides for proliferating cells, which subsequently activate the metabolizing enzymes of adenosinergic pathway. Depending on Ado receptor subtype expressed on cancer cells, Ado can either promotes cell proliferation, or can act as pro-apoptotic mediator, primarily through receptors A2A, A2B and A3 (Di Virgilio and Adinolfi, 2017; Muller-Haegele et al., 2014; Ohta, 2016).

Physiologically, ADA modulates T cells proliferation and differentiation (Hasko et al., 2018). It shows increased activity under nitrosative stress conditions where high levels of serum ADA were associated with metastatic lymph nodes and with proliferating cells in breast, gastric, bladder and colon cancers (Muller-Haegele et al., 2014; Ohta, 2016; Whiteside, 2017).

This study evaluated the serum levels of NO, MDA and $\mathrm{GSH}$, and $\mathrm{XO}$ and ADA activities, as markers of nitro-oxidative stress, in relation to vascularization of tumors at TNM stage IV in advanced gallbladder carcinoma.

\section{Materials and methods}

\section{Subjects and samples collection}

GBC patients (40) and healthy donors (40) enrolled in this study have approved and signed an informed consent according to the Code of Ethics of the World Medical Association (Declaration of Helsinki) for experiments involving Humans. Biochemical assays were approved by the local Ethical Committee of the "Université des Sciences et de la Technologie Houari Boumediene, Algiers, Algeria".
Biopsies (15) taken for the diagnosis of GBC patients were classified according to the tumor-node-metastasis (TNM) staging criteria (AJCC, 7th, 2010) (Byrd and Greene, 2018). The highest incidence of GBC was found in the age groups 50-60 (42.5\%) and 60-70 (35\%). The lowest incidence (2.5\%) was associated to the age groups 30-40 and >80 years. Women displayed higher prevalence for GBC (female to male ratio, $2.3: 1)$.

Since most of the patients were diagnosed at advanced stage of GBC, and as some of them had already been cholecystectomized, we selected biopsies at TNM stage IV instead of surgical specimens for this study.

Eighty venous blood were sampled from $40 \mathrm{GBC}$ patients of 38-83 years old (12 men and 28 women; mean age of $60 \pm$ 9 years), and 40 controls donors matching age (38-67 years; 10 men and 30 women). Sera were collected by centrifugation from venous blood, deproteinized with appropriate methods, and used for the determination of Ado catabolism and nitro-oxidative stress markers.

\section{Immunodetection of CD34 and determination of microvessel density}

The controls biopsies were collected from patients taken to surgery for laparoscopic cholecystectomy for suspected cholecystitis.

Specimen of freshly resected biopsies (from 15 GBC patients and 5 controls donors) were fixed in 10\% formalin phosphate buffered saline (PBS, $\mathrm{pH}$ 7.35), and processed for topographic histology. Sections $(4 \mu \mathrm{m})$ were mounted on gelatin-coated slides and incubated with CD34 primary antibodies (Abcam), overnight at $4{ }^{\circ} \mathrm{C}$. After washing, slides were incubated for $90 \mathrm{~min}$ at room temperature with the secondary antibody conjugated to horse radish peroxidase (HRP). Slides treated with 3 , 3-diaminobenzidine (DAB) and $\mathrm{H}_{2} \mathrm{O}_{2}$, were counter stained with Mayer's hematoxylin. Expression and distribution of CD34 were analyzed by light microscopy.

The microvessel density (MVD) was used as an index of angiogenesis (Marrogi et al., 2000). CD34 immunostained sections were screened at $\times 40$ magnification and the most vascularized areas were selected as hotspots, where tumor vessels were counted. CD34 immunohistochemical evaluation and scoring was semi quantitatively rated as follows: negative: $<5 \%$ immunostained cells $(-)$, positive: $>5 \%$ immunostained cells: (+); 5-10\% immunostained cells: $(++)$; >10\% immunostained cells: $(+++)$.

The mean value of CD34 positive microvessels was measured in 3-5 hot spots of CD34-positive cells and cell clusters. Tissue specimens were independently analyzed by 2-4 investigators.

\section{Determination of nitro-oxidative stress markers}

Serum redox status was evaluated by measuring the levels of malondialdehyde (MDA), an end product of lipid peroxidation (Draper et al., 1993), nitrite $\left(\mathrm{NO}_{2}^{-}\right)$, the stable end product of NO (Ding et al., 1988), and glutathione (GSH), an index of antioxidant potential (Ellman, 1959).

Sera were boiled in $0.8 \%(\mathrm{w} / \mathrm{v})$ 2-thiobarbituric acid mixture containing $8.1 \%(\mathrm{w} / \mathrm{v})$ SDS, $20 \%$ (v/v) acetic acid ( $\mathrm{pH} 3.5$ ), $1 \mathrm{~h}$ at $95{ }^{\circ} \mathrm{C}$. Supernatants were collected by centrifugation at $3000 \mathrm{~g}$ for $10 \mathrm{~min}$, and absorbance was read at $532 \mathrm{~nm}$. MDA levels were expressed in $\mu$ moles/l of thiobarbituric acid reactive substances.

Equal volumes of deproteinized sera and Griess reagent ( $1 \%$ sulphanilamide and $0.1 \% \mathrm{~N}$ - (1-napthyl) ethylene 
diamine dihydrochloride in $2.5 \% \mathrm{H}_{3} \mathrm{PO}_{4}$ ), were allowed to react, $20 \mathrm{~min}$ at room temperature. Absorbance was read at $540 \mathrm{~nm}$, and $\mathrm{NO}_{2}{ }^{-}$levels expressed in $\mu$ moles/l, using a sodium nitrite curve as standard.

Deproteinized sera were treated with Ellman's reagent ((5,5'-dithiobis (2-nitrobenzoic acid)) in $0.2 \mathrm{M}$ phosphate buffer, $\mathrm{pH}$ 8.0. Absorbance was read at $412 \mathrm{~nm}$, and GSH levels were expressed in $\mu$ moles/l using a standard GSH curve.

\section{Determination of adenosine deaminase and xanthine oxidase activities}

Serum adenosine deaminase (ADA) was determined by Bertholet reaction (Giusti, 1974). Samples were incubated with $0.5 \mathrm{mM}$ adenosine (Ado) in phosphate buffer $\mathrm{pH} \mathrm{6.5,} 1 \mathrm{~h}$ at $37{ }^{\circ} \mathrm{C}$. After stopping the reaction, absorbance was read at $620 \mathrm{~nm}$. The results were expressed in unit (U/1) of enzyme activity, which is defined as the amount of enzyme that releases $1 \mu$ mole of ammonia/min.

Xanthine oxidase (XO) activity was assayed by hypoxanthine oxidation method (Parks et al., 1988). Sera were incubated with $1.5 \mathrm{mM}$ xanthine, $5 \mathrm{~min}$ at $25^{\circ} \mathrm{C}$, and absorbance was read at $292 \mathrm{~nm}$. The results were expressed in unit enzyme/l, where one unit of $\mathrm{XO}$ is defined as the amount of enzyme that produces $1 \mu$ mole uric acid/ $\mathrm{min}$.

\section{Statistical analysis}

The results were expressed as mean \pm SD. Differences between controls and patients groups were analysed with the two-tailed non-parametric Mann-Whitney test, using $p<0.05$ for statistical significance. Data were analysed with GraphPad Software (Prism version 5.00, San Diego, CA, USA).

Correlations between biochemical parameters and MVD were evaluated by Sperman analyses. Diagnostic accuracy and cut-off values were determined by receiver operating characteristic (ROC) curve performed with SPSS program (IBM SPSS Statistics for Windows, Version 24.0. Armonk, NY: IBM Corp).
To determine the degree of association of each biochemical factor to gallbladder cancer or to MDV, a binary logistic regression and multiple regression, respectively were performed with GBC or MVD as dependent variables and MDA, GSH, iNOS, ADA and XO as independent variables (SPSS program).

\section{Results}

\section{General cohort characteristics}

Forty patients consisting of 12 males and 28 females with mean age of 60 (range: 38-83 years old). Forty individuals form the control group with 10 males and 30 females (range: 38-67 years old). The sample consisted of $40 \mathrm{GBC}$ patients and 40 healthy subjects were analysed for MDA, GSH and NO levels and XO and ADA activities. The metastases was observed in 22 of the 40 GBC patients and all the samples are adenocarcinomas.

Fifteen biopsies from GBC patients and five controls biopsies were taken for the CD34 expression scoring and MVD analysis.

\section{High serum MDA/GSH ratio is an index of severe toxicity in GBC patients}

Mean serum levels of MDA increased by 3.2-fold ( $p<0.001)$, whereas those of GSH decreased by $44.6 \%(p<0.001)$ in GBC patients, compared to healthy controls. The resulting MDA/ GSH ratio enhanced by 6.5 -fold $(p<0.001)$, indicating severe serum toxicity in advanced GBC (Figs $1 \mathrm{~A}-\mathrm{C}$ ). ROC curve for MDA and GSH, displayed high area under the curve (AUC; 0.974 and 0.930 ) with optimum cut-off points of $3.72 \mu \mathrm{mol} / 1$ and $36.91 \mu \mathrm{mol} / 1 ; 92.5 \%$ and $90 \%$ sensitivity; $95 \%$ and $80 \%$ specificity and $93.75 \%$ and $85 \%$ accuracy, respectively. The positive and negative likelihood ratios were 0.98 and 1.13 , and 0.96 and 1.11, respectively (Table 1; Figs 1D, 1E).
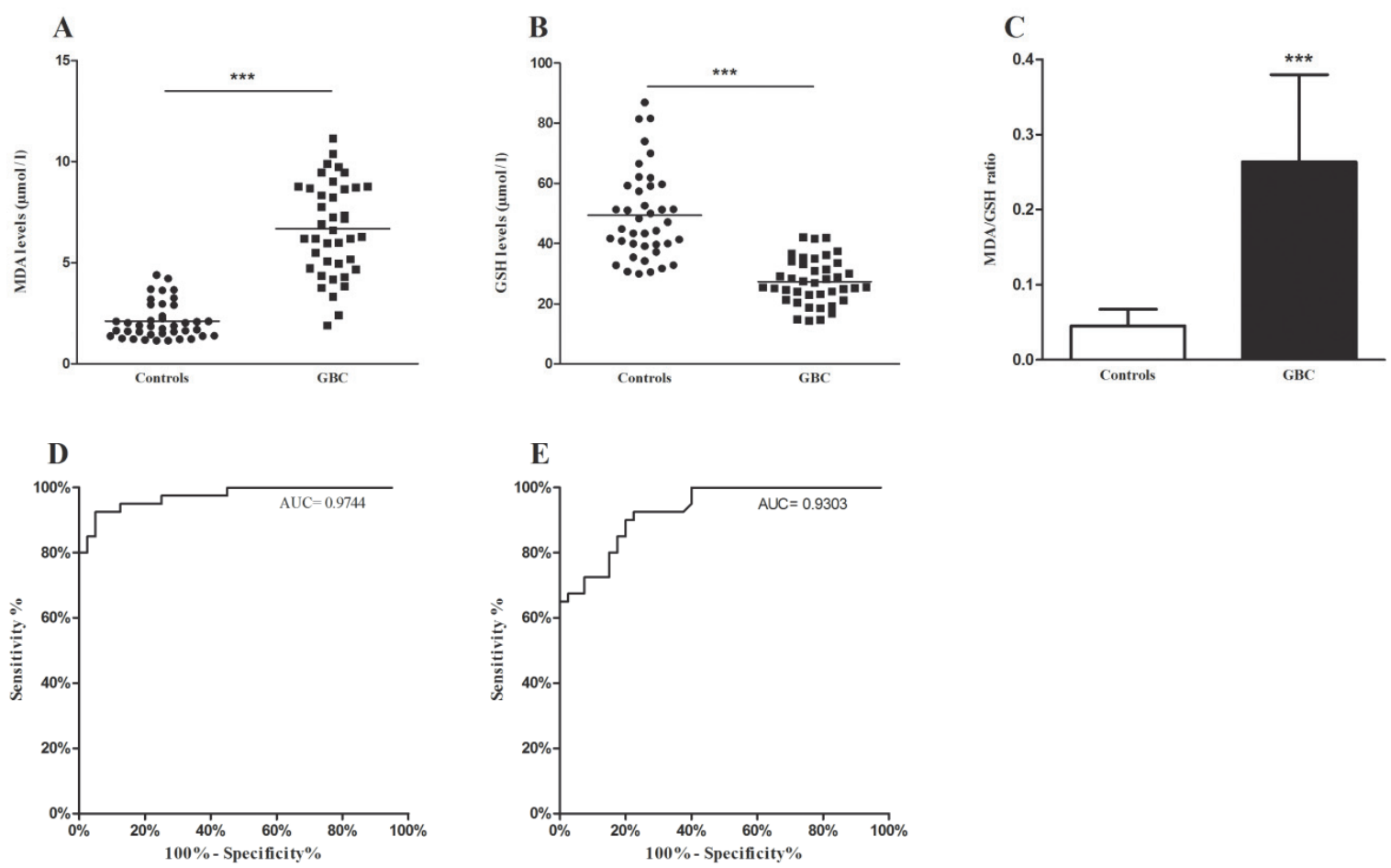

Fig. 1. Scattered dot plot showing mean level of MDA (A) and GSH (B), and MDA/GSH ratio (C) in sera from 40 patients with gallbladder carcinoma and from 40 healthy individuals used as controls. ROC curves for MDA (D) and GSH (E). AUC, area under the curve. ROC, receiver operating characteristic. 
Table 1. Parameters characteristics for MDA, GSH, ADA activity, XO activity and nitrites levels in 40 controls and 40 GBC patients. LR', LR', positive and negative Likelihood ratio

\begin{tabular}{|c|c|c|c|c|c|c|}
\hline Markers & Cut-off level & Specificity & Sensibility & Accuracy & $\mathrm{LR}^{+}$ & $\mathrm{LR}^{-}$ \\
\hline MDA & $3.72 \mu \mathrm{mol} / 1$ & $95 \%$ & $92.5 \%$ & $93.75 \%$ & 0.98 & 0.96 \\
\hline GSH & $36.91 \mu \mathrm{mol} / 1$ & $80 \%$ & $90 \%$ & $85 \%$ & 1.13 & 1.11 \\
\hline Nitrites & $21.21 \mu \mathrm{mol} / 1$ & $87.5 \%$ & $90 \%$ & $88.75 \%$ & 7.2 & 0.11 \\
\hline $\mathrm{ADA}$ & $17.02 \mathrm{U} / 1$ & $77.5 \%$ & $100 \%$ & $88.75 \%$ & 4.44 & 0.0 \\
\hline XO & $5.41 \mathrm{U} / 1$ & $80 \%$ & $60 \%$ & $70 \%$ & 0.75 & 0.73 \\
\hline
\end{tabular}

\section{Increased serum iNOS activity in GBC patients}

Mean serum level of nitrites enhanced by 2 -fold $(p<0.001)$ in GBC patients, compared to healthy individuals (Fig. 2A). ROC curve showed high AUC (0.962), an optimum cut-off point of
$21.21 \mu \mathrm{mol} / \mathrm{l} ; 90 \%$ sensitivity, $87.5 \%$ specificity and $88.75 \%$ accuracy. The positive and negative likelihood ratios were 7.2 and 0.11 , respectively (Table 1; Fig. 2B).

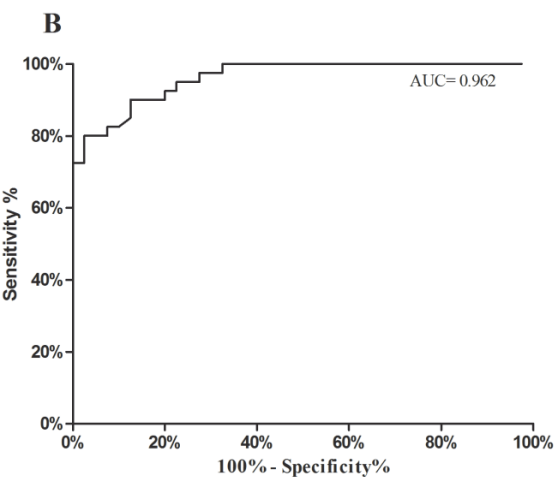

Fig. 2. Scattered dot plot showing mean level of serum nitrites in sera from 40 patients with gallbladder carcinoma and from 40 controls (A). ROC curve for nitrites levels $(\mathbf{B})$.

\section{Increased serum ADA activity in GBC patients}

Mean serum ADA activity increased by 2.69 -fold $(p<0.001)$ in GBC patients, compared to healthy controls (Fig. 3A). AUC of 0.948 and optimal cut-off point of $17.02 \mathrm{U} / 1$ provided the
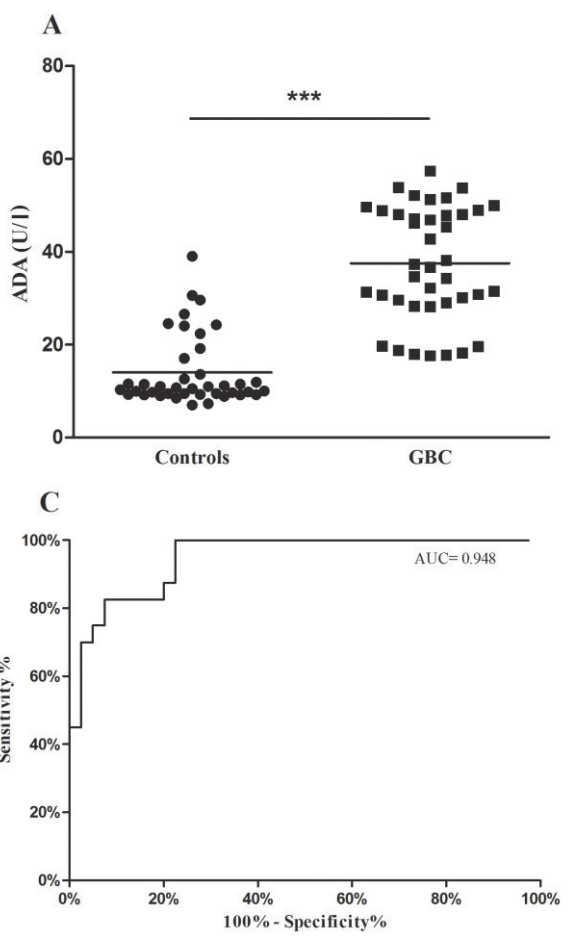

highest sum of sensitivity (100\%) and specificity (77.5\%). Diagnosis accuracy for ADA was $88.75 \%$. The positive and negative likelihood ratios were 4.4 and 0.0 , respectively (Table 1 ; Fig. 3C).
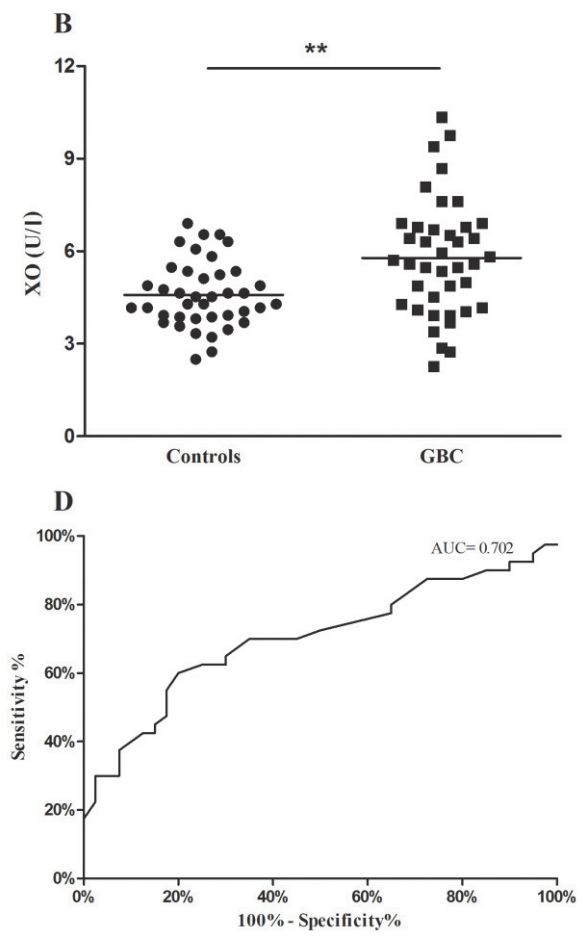

Fig. 3. Scattered dot plot showing mean level of serum ADA $(\mathbf{A})$ and XO activities $(\mathbf{B})$ in sera from 40 patients with gallbladder carcinoma and from 40 controls. ROC curve for ADA (C) and XO activities (D). 


\section{Increased serum $X O$ activity in $G B C$ patients}

Compared to healthy controls, mean serum XO activity enhanced by $21 \%(p<0.01)$ in GBC patients (Fig. 3B), with an AUC of 0.702 . The optimal cut-off point was $5.41 \mathrm{U} / 1$, with $60 \%$ sensitivity, $80 \%$ specificity, and $70 \%$ accuracy. The positive and negative likelihood ratios for $\mathrm{XO}$ activity were 0.75 and 0.73 , respectively (Table 1; Fig. 3D).

\section{CD34 expression and microvessel density increased in GBC biopsies}

Hematoxylin and eosin (H\&E)-stained sections of gallbladder tumors (Figs 4C, 4D) and controls (Figs 4A, 4B) were reviewed for tumor diagnosis and classification (Byrd and Greene, 2018). All the 15 biopsies were CD34 positives, with intermediate (10) to high (1) MVD grades. Neoplastic stroma showed dark brown spots of CD34 positive immuno-reactivity of newly formed micro-vasculatures (Figs $4 \mathrm{G}, 4 \mathrm{H}$ ), compared to normal gallbladder (Figs 4E, 4F).

The mean MVD in GBC peritumor tissue enhanced by 2.21-fold (23.02 \pm 5.72 microvessels/ field, $p<0.01)$, compared to normal gallbladder tissue, and the median MVD was 25 (range 10-30) microvessels/ field (Fig. 5A).
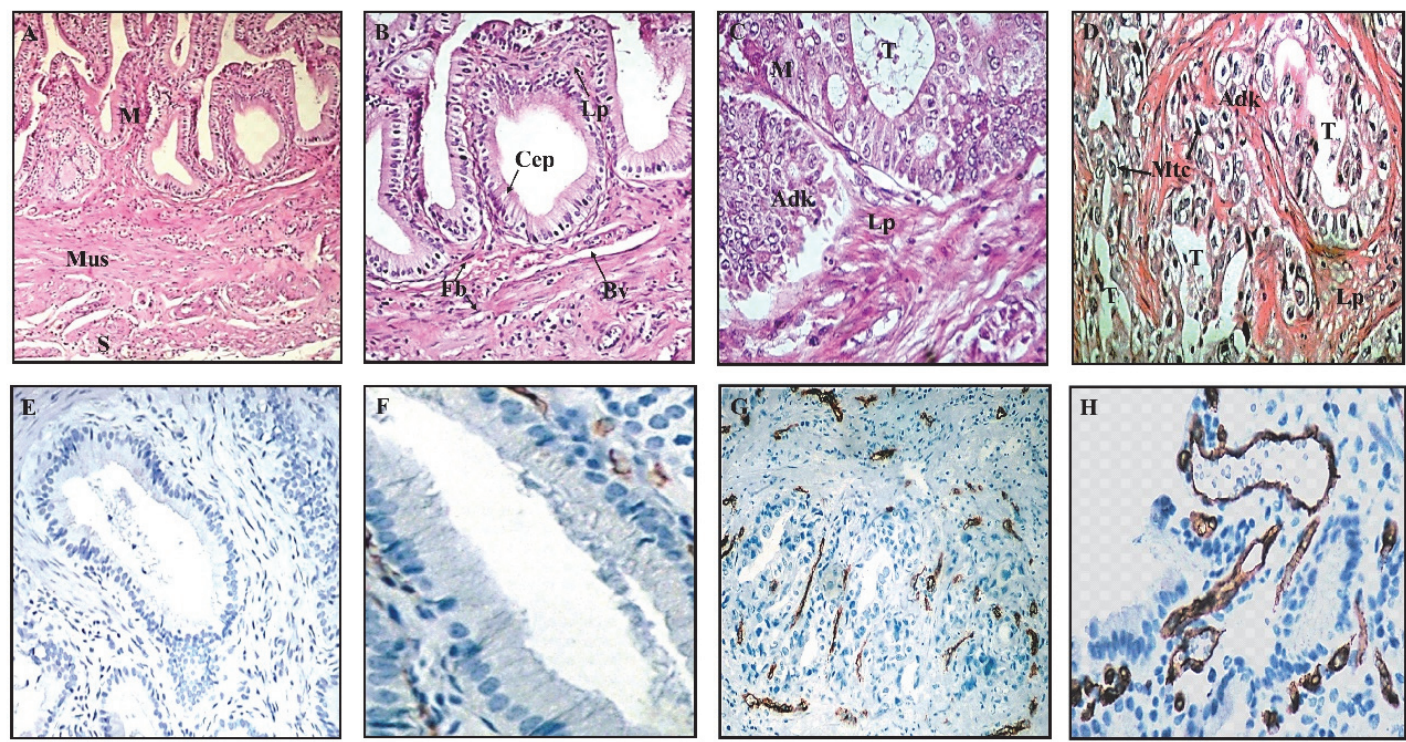

Fig. 4. Micrographs of H\&E-stained sections of control gallbladder (A, G × 200; B, Gr × 400); GBC H\&E (C, G × 200; D, G × 400); CD34 immunostainning in control (E, G × 200; F, Gr × 400) and MVD in GBC tissue $(\mathbf{G}, \mathrm{G} \times 200 ; \mathbf{H}, \mathrm{Gr} \times 400)$. Adk, Adenocarcinoma; Bv, Blood vessels; Cep, Columnar epithelium; Fb, Fibroblasts; H\&E, Hematoxylin and Eosin; Lp, Lamina propria; Mtc, Mitotic cells; M, Mucosa; Mus, Muscular; S, Serous; T, Tubular structure.
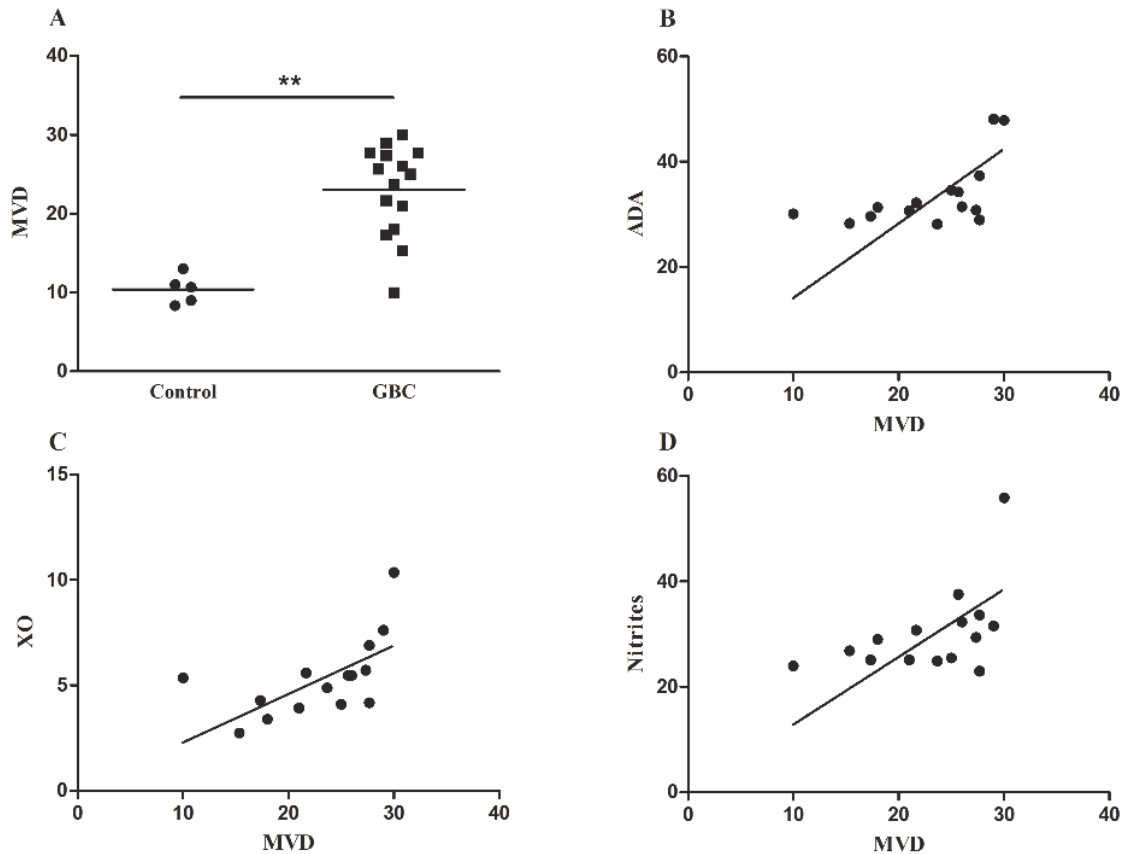

Fig. 5. Scatter dot plot of MVD in 5 normal gallbladder and 15 gallbladder adenocarcinoma (A). Spearman correlation analysis between sera ADA and XO and iNOS and MVD in 15 GBC patients. ADA vs. MVD (B); XO vs. MVD (C); Nitrites vs. MVD (D). 


\section{Correlation between biochemical parameters and microvessel density}

Spearman coefficient showed a positive correlation between MVD and serum XO activity $(r=0.7030, p<0.01)$, followed by ADA activity $(r=0.6148, p<0.05)$, and nitrites levels $(r=0.5438, p<0.05)$, in 15 GBC patients (Figs 5B-D). No relationship was found between MVD, and MDA or GSH. In addition, more than $91 \%$ (10/11) of CD34 positive GBC biopsies had intermediate MVD levels. Among them, 82\% (9/11) were associated with intermediate (6) to high (2) ADA and (1) XO activities, and $40 \%$ with intermediate (4) or high (1) iNOS activity. Importantly, in one tumor high MVD levels correlated to high ADA, XO and iNOS activities.

Multiple regression analysis of biochemical parameters (as independent variables) and MVD (as dependent variable) showed that ADA $(r=0.604, p=0.007)$ correlate to enhanced MVD (Table 2).

\section{Correlation between biochemical parameters and GBC progression}

A positive correlation was shown between serum ADA activity and nitrites levels $(r=0.3419, p<0.05)$, and between nitrites and MDA levels $(r=0.3775, p<0.05)$ in GBC patients (Fig. 6). Moreover, XO and ADA activity $(r=0.5487, p<0.001)$ correlated positively, whereas GSH was inversely correlated $(r=0.3197, p<0.05)$ with nitrites levels. There was no significant correlation between sex and iNOS, ADA or XO activities.

Table 2. Multiple regression analysis for biochemical parameters determinants of MVD in 15 GBC patients

\begin{tabular}{lccc}
\hline Variables & Regression coefficient & Standard error & $P$ value \\
\hline MDA & -0.173 & 0.499 & 0.383 \\
GSH & -0.135 & 0.075 & 0.417 \\
Nitrites & 0.404 & 0.141 & 0.082 \\
ADA & 0.604 & 0.092 & 0.007 \\
XO & -0.077 & 0.647 & 0.593
\end{tabular}

The enter method is applied to this model with MVD as a dependent variable, and MDA, GSH, nitrites, ADA and XO as independent variables simultaneously. Values are adjusted for all variables in table and $p$ value for each variable are displayed.
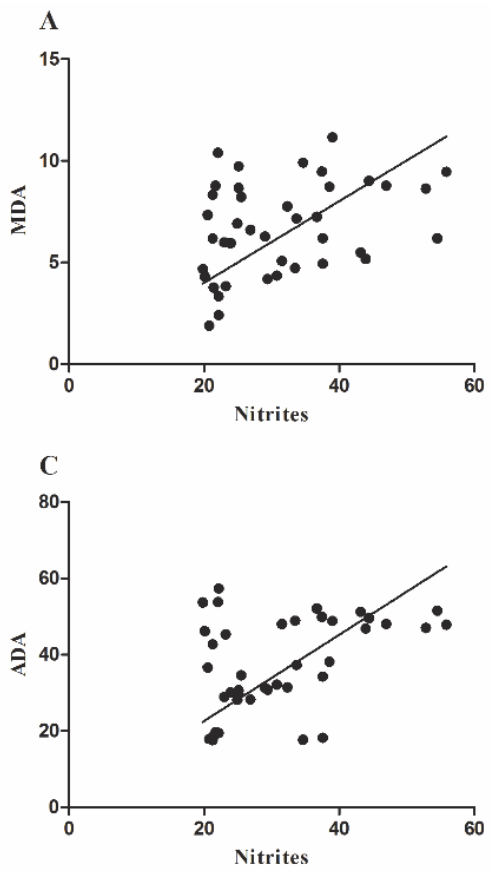
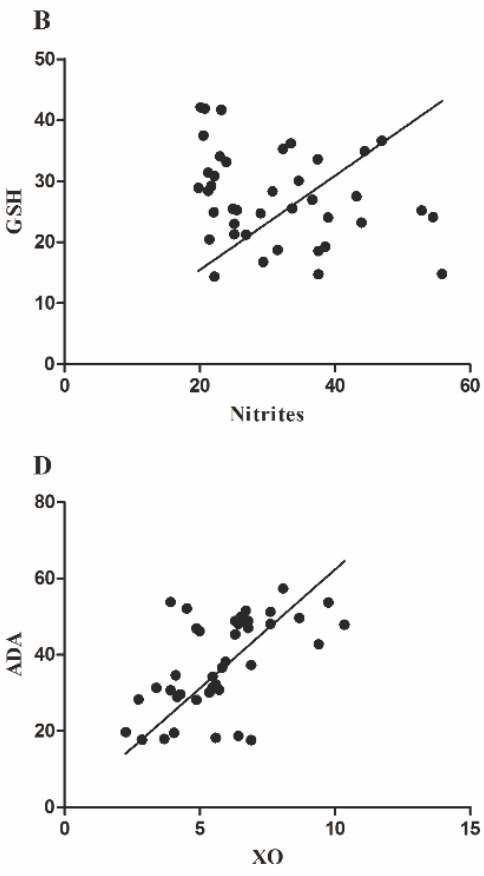

Fig. 6. Scattered dot plot and spearman correlation analysis between sera biochemical parameters in 40 GBC patients. MDA vs. iNOS (A); GSH vs. iNOS (B); ADA vs. iNOS (C); ADA vs. XO (D).

Binary logistic regression analysis examining the independent risk predictors for GBC malignancy indicated that NO derived-iNOS (OR $=1.93,95 \%$ CI: $0.78-4.80, p>0.05)$ and GSH (OR $=0.84,95 \%$ CI: 0.67-1.04, $p>0.05)$ were not associated with GBC risk (Table 3). Whereas, there is a significant trend of association between GBC and ADA (OR $=1.28,95 \%$ CI: $1.13-1.44, p<0.001)$ and XO (OR $=2.81,95 \%$ CI: 1.39 5.66, $p<0.05)$. Importantly, high MDA level was significantly associated with an increased risk of $\mathrm{GBC}(\mathrm{OR}=5.78,95 \% \mathrm{CI}$ : 1.15-29.02, $p<0.05)$. 
Table 3. Independent risk predictors of gallbladder carcinoma, defined by binary logistic regression analysis considering MDA, $\mathrm{GSH}$, nitrites, ADA and $\mathrm{XO}$ in $40 \mathrm{GBC}$ patients

\begin{tabular}{lccc}
\hline Variables & Adjusted odds ratio & $\begin{array}{c}95 \% \text { CI for } \\
\text { odds }\end{array}$ & P value \\
\hline MDA & 5.782 & $1.152-29.029$ & 0.033 \\
GSH & 0.840 & $0.675-1.046$ & 0.120 \\
Nitrites & 1.937 & $0.782-4.801$ & 0.153 \\
ADA & 1.284 & $1.137-1.449$ & $<0.001$ \\
XO & 2.811 & $1.395-5.662$ & 0.02 \\
\hline
\end{tabular}

The enter method is applied to this model with gallbladder carcinoma as a dependent variable, and MDA, GSH, nitrites, ADA and $\mathrm{XO}$ as independent variables. The adjusted odds ratio, 95\% confidence interval, and $p$ value for each variable are displayed.

\section{Discussion}

This study evaluated the potential correlation between serum redox status, oxidative Ado catabolism and MVD in advanced gallbladder carcinoma.

The results revealed sustained serum nitro-oxidative stress and purine catabolism supported by the 2.0, 2.69 and 1.26 -fold increase in mean level of nitrites, ADA and XO activities (Figs 2, 3). Although our cohort of patients is rather small ( $n=40<100)$, it showed a prevalence of GBC in women (female to male ratio, $2.3: 1$ ), but no significant correlation between sex and iNOS, ADA or XO activities.

The up expression of CD34 in all biopsies at TNM stage IV ( $n=15)$, resulted in 2.2-fold enhanced MVD, which correlates positively to $\mathrm{XO}$ activity, followed by ADA and iNOS activities $(r=0.7030, r=0.6148, r=0.5438)$. This suggests that under chronic nitro-oxidative stress, sustained purine salvage pathway could enriched developing solid tumors in new vasculatures. Persistent flow of reactive oxygen and nitrogen species (ROS) supplied by the cross talk between iNOS, ADA and XO activities can act as a strong accelerator for GBC progression (Cipak et al., 2017; Espinoza et al., 2016; Waris and Ahsan, 2006).

Tumor aggressiveness was associated with iNOS expression in two-thirds of GBC cases, whereas the link between iNOS and angiogenesis is quite weak in our small cohort of GBC patients (Table 2) (Niu et al., 2004; Thompson et al., 2015; Zhang et al., 2003). TNF-a, a well known stimulant of iNOS activity and a potent inducer of iNOS expression, was shown to promote gallbladder cancer growth, lymphangiogenesis and invasion (Du et al., 2014; Niu et al., 2004; Zhang et al., 2003). Otherwise, the drastic systemic toxicity indicated by the 6.5-fold increase in serum MDA/GSH ratio, durably settled in patients with GBC at TNM stage IV, as a result of GSH depletion (45\%) and a burst in lipid peroxidation (MDA, 3.2-fold) (Fig. 1; Table 1). GSH plays a critical role in controlling ROS accumulation by enzymatic conjugation and/or elimination of lipids peroxides (Ramsay and Dilda, 2014). Indeed, sustained oxidative chain reactions of polyinsaturated fatty acids, leads to the release of volatile electrophiles (HNE, MDA...), which bind to proteins and DNA, forming covalents oxidized adducts. HNE was characterised as pro-apoptotic and oxidative stress-signaling electrophile (Gasparovic et al., 2017; Zhong and Yin, 2015). Moreover, the HNE-dG adduct was shown to be strongly genotoxic and mutagenic for DNA of various origin, including mitochondrial DNA (Zhong and Yin, 2015).
GBC is the most aggressive malignancy of biliary tract. Due to the silent and rapid expansion of tumor, most patients are diagnosed at advanced stage, resulting in limited effectiveness of chemotherapy, and a low impact on overall survival (Ferlay et al., 2015; Hundal and Shaffer, 2014; Sharma et al., 2017; Shukla et al., 2018).

Multiple regression analysis showed that ADA $(r=0.604)$ could sustain MVD development. More, multivariate analysis of biochemical markers by binary logistic regression suggests that $\mathrm{ADA}(\mathrm{OR}=1.28,95 \% \mathrm{CI}: 1.13-1.44), \mathrm{XO}(\mathrm{OR}=2.81,95 \%$ CI: $1.39-5.66)$ and MDA (OR $=5.78,95 \%$ CI: $1.15-29.02)$ could be potential predictive factors for GBC, whereas GSH (OR $=0.84,95 \%$ CI: 0.67-1.04) was inversely associated to GBC (Table 3). Although higher positive correlation was shown between ADA and XO activities $(r=0.5487, p<0.001)$, XO activity was less enhanced, compared to ADA activity (2.69 vs. 1.26-fold). Under hypoxic conditions, XO contributes to oncogenesis by the metabolic activation of carcinogenic mediators and/or through XO-derived ROS (Battelli et al., 2016).

Ado catabolism through salvage pathway remains unexplored in GBC. ADA is released by infected phagocytes, during immune response to tuberculosis, and was extensively used for the diagnosis of tuberculous pleural effusion, and patients with lymphoma (Łupicka-Słowik et al., 2018; Yao et al., 2014). ADA is stimulated by cytokines and growth factors (IL-2, IL-12 and INF- $\gamma$ ) released by actively proliferating tissues (Quail and Joyce, 2013) and considered as inflammation marker during hypoxia (Castilhos et al., 2018). In concert, the mobilization of Ado receptors $\mathrm{A} 2 \mathrm{~A}$ and $\mathrm{A} 2 \mathrm{~B}$ on endothelial cells enhances the production of pro-angiogenic factors mediated by hypoxia-inducible factor (HIF)-1a including VEGF (vascular endothelial growth factor) and IL-8 (Quail and Joyce, 2013).

By driving tumor escape from the immune control and down regulating tissue-destructive effects of activated immune cells, Ado finely tunes the growth and dissemination of tumor mass through the engagement of adenosinergic receptors expressed on neoplastic cells (Moreno et al., 2018; Öztürk et al., 2015). Ado also mediates the inhibition of effector immune cells, including CD8 cytotoxic T lymphocytes, natural killer cells, dendritic cells, macrophages, and promotes $\mathrm{CD}^{+}$cells differentiation into $\mathrm{T}$ regulatory cells (Chaudhary and Elkord, 2016). Angiogenesis and IL-17 are positively correlated in human gastric, colorectal and hepatocellular tumors (Yang et al., 2014). Th17 cells and their associated cytokines, mainly IL-17A can mediate tumor growth by stimulating VEGF production and suppressing anti-tumor immunity ( $\mathrm{Wu}$ et al., 2016). The resistance of Th17 cells to Ado correlates to enhanced Ado clearance by ADA (Longhi et al., 2014; Yang et al., 2014).

\section{Conclusions}

To date, the complete resection of the gallbladder still remains the only curative approach. In conclusion, although our cohort of $\mathrm{GBC}$ patients is quite small and the biochemical analyzes carried out exclusively on TNM stage IV, our results support the association between oxidative catabolism of Ado by ADA and $\mathrm{XO}$ and accumulation of the lipid peroxides marker, MDA along with decreased GSH levels. Therefore, ADA activity may play a key role in the pathogenesis of GBC and tumor angiogenesis.

The progress in deciphering the molecular alterations supporting the progression of gallbladder cancer could favour the emergence of more effective prognostic and curative strategies. 


\section{Conflict of interests}

The authors declare that they have no conflict of interests.

\section{Funding}

This research did not receive any specific grant from funding agencies in the public, commercial or not-for-profit sectors.

\section{Acknowledgement}

Authors warmly thank all the Patients and Volunteers involved in this study.

All authors contributed equally to the design, results analyses and to manuscript editing.

\section{References}

Battelli MG, Polito L, Bortolotti M, Bolognesi A (2016). Xanthine oxidoreductase in cancer: more than a differentiation marker. Cancer Med 5(3): 546-557. DOI: 10.1002/cam4.601.

Byrd DR, Greene FL (2018). The eighth edition of TNM: Implications for the surgical oncologist. Ann Surg Oncol 25(1): 10-12. DOI: 10.1245/s10434-017-6027-8.

Castilhos LG, Adefegha SA, Doleski PH, Bertoldo TM, Moritz CE, Casali EA, Leal DB (2018). NTPDase, 5'-nucleotidase and adenosine deaminase activities and purine levels in serum of sickle cell anemia patients. J Appl Biomed 16: 208-213. DOI: 10.1016/j. jab.2017.12.004.

Chaudhary B, Elkord E (2016). Regulatory T cells in the tumor microenvironment and cancer progression: role and therapeutic targeting. Vaccines (Basel) 4(3): E28. DOI: 10.3390/ vaccines 4030028.

Cipak Gasparovic A, Zarkovic N, Zarkovic K, Semen K, Kaminskyy D, Yelisyeyeva O, Bottari SP (2017). Biomarkers of oxidative and nitro-oxidative stress: conventional and novel approaches. Br J Pharmacol 174(12): 1771-1783. DOI: 10.1111/bph.13673.

De Oliveira GA, Cheng RY, Ridnour LA, Basudhar D, Somasundaram V, McVicar DW, et al. (2017). Inducible nitric oxide synthase in the carcinogenesis of gastrointestinal cancers. Antioxid Redox Signal 26(18): 1059-1077. DOI: 10.1089/ ars.2016.6850.

Di Virgilio F, Adinolfi E (2017). Extracellular purines, purinergic receptors and tumor growth. Oncogene 36(3): 293-303. DOI: 10.1038/onc.2016.206.

Ding AH, Nathan CF, Stuehr DJ (1988). Release of reactive nitrogen intermediates and reactive oxygen intermediates from mouse peritoneal macrophages. Comparison of activating cytokines and evidence for independent production. J Immunol 141(7): 2407-2412.

Draper H, Squires E, Mahmoodi H, Wu J, Agarwal S, Hadley M (1993). A comparative evaluation of thiobarbituric acid methods for the determination of malondialdehyde in biological materials. Free Radic Biol Med 15(4): 353-363. DOI: 10.1016/08915849(93)90035-s.

Du Q, Jiang L, Wang X, Wang M, She F, Chen Y (2014). Tumor necrosis factor- $\alpha$ promotes the lymphangiogenesis of gallbladder carcinoma through nuclear factor-kB-mediated upregulation of vascular endothelial growth factor-C. Cancer Sci 105(10): 1261-1271. DOI: 10.1111/cas.12504.

Ellman GL (1959). Tissue sulfhydryl groups. Arch Biochem Biophys 82(1): 70-77. DOI: 10.1016/0003-9861(59)90090-6.

Espinoza JA, Bizama C, Garcia P, Ferreccio C, Javle M, Miquel JF, et al. (2016). The inflammatory inception of gallbladder cancer. Biochim Biophys Acta 1865(2): 245-254. DOI: 10.1016/j. bbcan.2016.03.004

Ferlay J, Soerjomataram I, Dikshit R, Eser S, Mathers C, Rebelo M, et al. (2015). Cancer incidence and mortality worldwide: sources, methods and major patterns in GLOBOCAN 2012. Int J Cancer 136(5): E359-E386. DOI: 10.1002/ijc.29210.
Gasparovic AC, Milkovic L, Sunjic SB, Zarkovic N (2017). Cancer growth regulation by 4-hydroxynonenal. Free Radic Biol Med 111: 226-234. DOI: 10.1016/j.freeradbiomed.2017.01.030.

Giusti G (1974). Adenosine deaminase, Methods of Enzymatic Analysis (Second Edition), Volume 2. Elsevier, pp. 1092-1099. DOI: 10.1016/B978-0-12-091302-2.X5001-4.

Hasko G, Antonioli L, Cronstein BN (2018). Adenosine metabolism, immunity and joint health. Biochem Pharmacol 151: 307-313. DOI: 10.1016/j.bcp.2018.02.002.

Hundal R, Shaffer EA (2014). Gallbladder cancer: epidemiology and outcome. Clin Epidemiol 6: 99-109. DOI: 10.2147/CLEP.S37357.

Kepp O, Loos F, Liu P, Kroemer G (2017). Extracellular nucleosides and nucleotides as immunomodulators. Immunol Rev 280(1) 83-92. DOI: 10.1111/imr.12571.

Longhi MS, Moss A, Bai A, Wu Y, Huang H, Cheifetz A, et al. (2014). Characterization of human CD39+ Th17 cells with suppressor activity and modulation in inflammatory bowel disease. PloS One 9(2): e87956. DOI: 10.1371/journal.pone.0087956.

Łupicka-Słowik A, Psurski M, Grzywa R, Bobrek K, Smok P, Walczak M, et al. (2018). Development of adenosine deaminasespecific IgY antibodies: diagnostic and inhibitory application. Appl Biochem Biotechnol 184(4): 1358-1374. DOI: 10.1007/s12010017-2626-x.

Marrogi AJ, Travis WD, Welsh JA, Khan MA, Rahim H, Tazelaar H, et al. (2000). Nitric oxide synthase, cyclooxygenase 2, and vascular endothelial growth factor in the angiogenesis of non-small cell lung carcinoma. Clin Cancer Res 6(12): 4739-4744.

Moreno E, Canet J, Gracia E, Lluís C, Mallol J, Canela EI, et al. (2018). Molecular Evidence of Adenosine Deaminase Linking Adenosine A2A Receptor and CD26 Proteins. Front Pharmacol 9: 106. DOI: $10.3389 /$ fphar.2018.00106.

Muller-Haegele S, Muller L, Whiteside TL (2014). Immunoregulatory activity of adenosine and its role in human cancer progression. Expert Rev Clin Immunol 10(7): 897-914. DOI: 10.1586/1744666X.2014.915739.

Niu XJ, Wang ZR, Wu SL, Geng ZM, Zhang YF, Qing XL (2004). Relationship between inducible nitric oxide synthase expression and angiogenesis in primary gallbladder carcinoma tissue. World J Gastroenterol 10(5): 725-728. DOI:10.3748/wjg.v10.i5.725.

Ohta A (2016). A metabolic immune checkpoint: adenosine in tumor microenvironment. Front Immunol 7: 109. DOI: 10.3389/ fimmu.2016.00109.

Öztürk B, Kocaoğlu EH, Durak ZE (2015). Effects of aqueous extract from Silybum marianum on adenosine deaminase activity in cancerous and noncancerous human gastric and colon tissues. Pharmacogn Mag 11(41): 143-146. DOI: 10.4103/09731296.149729.

Parks DA, Williams TK, Beckman JS (1988). Conversion of xanthine dehydrogenase to oxidase in ischemic rat intestine: a reevaluation. Am J Physiol 254(5 Pt 1): G768-G774. DOI: 10.1152/ ajpgi.1988.254.5.G768.

Quail DF, Joyce JA (2013). Microenvironmental regulation of tumor progression and metastasis. Nat Med 19(11): 1423-1437. DOI: $10.1038 / \mathrm{nm} .3394$.

Ramsay EE, Dilda PJ (2014). Glutathione S-conjugates as prodrugs to target drug-resistant tumors. Front Pharmacol 5: 181. DOI: 10.3389/fphar.2014.00181.

Sharma A, Sharma KL, Gupta A, Yadav A, Kumar A (2017). Gallbladder cancer epidemiology, pathogenesis and molecular genetics: Recent update. World J Gastroenterol 23(22): 39783998. DOI: 10.3748/wjg.v23.i22.3978.

Shukla SK, Singh G, Shahi K, Bhuvan, Pant P (2018). Staging, treatment, and future approaches of gallbladder carcinoma. J Gastrointest Cancer 49(1): 9-15. DOI: 10.1007/s12029-0170036-5.

Singhal SS, Singh SP, Singhal P, Horne D, Singhal J, Awasthi S (2015). Antioxidant role of glutathione S-transferases: 4-Hydroxynonenal, a key molecule in stress-mediated signaling. Toxicol Appl Pharmacol 289(3): 361-370. DOI: 10.1016/j.taap.2015.10.006.

Tekcham DS, Tiwari PK (2016). Epigenetic regulation in gallbladder cancer: Promoter methylation profiling as emergent novel biomarkers. Asia Pac J Clin Oncol 12(4): 332-348. DOI: 10.1111/ ajco.12507. 
Thompson PA, Khatami M, Baglole CJ, Sun J, Harris SA, Moon EY, et al. (2015). Environmental immune disruptors, inflammation and cancer risk. Carcinogenesis 36(Suppl. 1): S232-S253. DOI: $10.1093 /$ carcin/bgv038.

Waris G, Ahsan H (2006). Reactive oxygen species: role in the development of cancer and various chronic conditions. J Carcinog 5: 14. DOI: 10.1186/1477-3163-5-14.

Whiteside TL (2017). Targeting adenosine in cancer immunotherapy: a review of recent progress. Expert Rev Anticancer Ther 17(6): 527-535. DOI: 10.1080/14737140.2017.1316197.

Wu X, Yang T, Liu X, Guo JN, Xie T, Ding Y, et al. (2016). IL-17 promotes tumor angiogenesis through Stat3 pathway mediated upregulation of VEGF in gastric cancer. Tumor Biol 37(4): 5493-5501. DOI: 10.1007/s13277-015-4372-4.
Yang B, Kang H, Fung A, Zhao H, Wang T, Ma D (2014). The role of interleukin 17 in tumour proliferation, angiogenesis, and metastasis. Mediators Inflamm 2014: 623759. DOI: 10.1155/2014/623759.

Yao CW, Wu BR, Huang KY, Chen HJ (2014). Adenosine deaminase activity in pleural effusions of lymphoma patients. QJM-INT J Med 107(11): 887-893. DOI: 10.1093/qjmed/hcu106.

Zhang M, Pan JW, Ren TR, Zhu YF, Han YJ, Kühnel W (2003). Correlated expression of inducible nitric oxide synthase and P53, Bax in benign and malignant diseased gallbladder. Ann Anat 185(6): 549-554. DOI: 10.1016/S0940-9602(03)80125-5.

Zhong H, Yin H (2015). Role of lipid peroxidation derived 4-hydroxynonenal (4-HNE) in cancer: focusing on mitochondria. Redox Biol 4: 193-199. DOI: 10.1016/j.redox.2014.12.011. 